\title{
Clinical Profile and Outcome of Childhood Autoimmune Hemolytic Anemia: A Single Center Study
}

\author{
Kasi Bharathi Thatikonda, Manas Kalra, Arun Danewa, Pallavi Sachdeva, Tanusree Paul, \\ Divij Sachdeva, Anupam Sachdeva \\ From Department of Pediatric Hematology Oncology and BMT, Institute of Child Health, Sir Ganga Ram Hospital, New Delhi.
}

Correspondence to: Dr Anupam Sachdeva, Director Pediatric Hematology Oncology and BMT, Institute for Child Health, Sir Ganga Ram Hospital, New Delhi. anupamace@yahoo.co.in Received: May 16, 2020; Initial review: August 01, 2020; Accepted: December 13, 2020.

\begin{abstract}
Objective: To analyze clinical and laboratory parameters, and treatment outcomes of children with autoimmune hemolytic anemia (AlHA). Methods: Retrospective analysis of 50 children aged 0-18 years. Monospecific direct antiglobulin test (DAT) and investigations for secondary causes were performed. Disease status was categorized based on Cerevance criteria. Results: Median (range) age at diagnosis was 36 (1.5-204) months. AlHA was categorized as cold (lgM+,C3+/cold agglutinin +$)(35 \%)$, warm $(\operatorname{lgG}+$ with/without $\mathrm{C} 3+)$ $(28 \%)$, mixed (IgG+, IgM+, C3+) (15\%) and paroxysmal cold hemoglobinuria (4\%). Primary AlHA accounted for $64 \%$ cases. Treatment modalities included steroid $(66 \%)$, intravenous immunoglobulin (IVIg) (4\%), steroid+IVIg (4\%), and steroid+rituximab (4\%). Treatment duration was longer for secondary AlHA than primary (11 vs 6.6 months, $P<0.02)$ and in patients needing polytherapy than steroids only ( 13.3 vs 7.5 months, $P<0.006)$. During median (range) follow-up period of 73 (1-150) months, 29 (58\%) remained in continuous complete remission, $16(32 \%)$ remained in complete remission. Conclusion: Infants with AlHA have a more severe presentation. Monospecific DAT and a thorough search for an underlying cause help optimize therapy in most patients of AlHA.
\end{abstract}

Keywords: Cerevance criteria, Direct antiglobulin test, Rituximab, Treatment.
A utoimmune hemolytic anemia (AIHA) is caused by the presence of auto-antibodies directed against antigens on the surface of red blood cells, leading to premature destruction $[1,2]$. AIHA is the main cause of acquired extra corpuscular hemolysis in children [1]. AIHA can be subdivided into primary (or idiopathic) and secondary. AIHA presenting with thrombocytopenia (Evans synd-rome) tends to have a more chronic and relapsing clinical course [3-5]. There is scarcity of data on Indian children with AIHA and their treatment outcome. We present data on children with AIHA from a single center in India.

\section{METHODS}

This is a retrospective analysis of data from January, 2007 to April, 2019 from our unit's database. Fifty children less than 18 years of age diagnosed with AIHA were enrolled in the study. AIHA was diagnosed based on the clinical presentation, positive direct anti globulin test (DAT) and at least one of the following: reticulocytosis, haptoglobin $<10$ $\mathrm{mg} / \mathrm{dL}$, and total bilirubin $>1 \mathrm{mg} / \mathrm{dL}$ [7]. DAT test was done by gel card method (Bio-Rad). Infants were evaluated for TORCH profile. Based on clinical suspicion, hepatitis B, hepatitis C, HIV serology, Epstein-Barr virus (EBV) PCR, cytomegalovirus (CMV) PCR, Mycoplasma pneumoniae antibody test and antinuclear antibody (ANA) were done. For children with repeated infections, immunoglobulin profile, Lymphocyte subset analysis (CD3, CD4, CD8, CD19, CD16, CD56) was performed. Monospecific DAT test routinely was performed for all children with suspected AIHA after the year 2013. AIHA was categorized based on types of serological antibodies (IgG, IgM, IgA, C3, and/or combinations) found in monospecific DAT test, cold agglutinin test and Donath Landsteiner test. History of drug intake and recent blood transfusion was obtained for all patients.

Glucocorticoids were used as the first line therapy in both warm and cold AIHA. In cold AIHA, additionally, treatment of the underlying disease was prioritized. The patient was kept warm and in cases with very severe anemia, packed red cell transfusion was given with a heat generator inside the tubing. Intravenous methylprednisolone $(2 \mathrm{mg} / \mathrm{kg} 8$ hourly for 3 days followed by oral prednisolone, $2 \mathrm{mg} / \mathrm{kg} /$ day for 4 weeks, then tapered gradually) was used for patients who were sick, unable to take orally or had very severe hemolysis. 
If there was complete remission after 4 weeks, tapering of prednisolone was done by $10 \%$ with each dose change over a period of 6 months. If there was no remission/ steroid dependence with prednisolone dose of $0.2 \mathrm{mg} / \mathrm{kg} / \mathrm{day}$, second line treatment was used. Secondline therapy comprised of either intravenous immunoglobulin (IVIg), rituximab, cyclosporine, mycophenolate mofetil (MMF) or azathioprine. In steroid dependent cases, one of the immunosuppressants (cyclosporine, MMF, azathioprine) was used. In common variable immunodeficiency (CVID), IVIg was additionally used. Packed red blood cell transfusion as supportive therapy was given if the child had a hemoglobin value less than 3 $\mathrm{g} / \mathrm{dL}$ or $3-6 \mathrm{~g} / \mathrm{dL}$ with cardiac failure or respiratory distress and needing intensive care unit (ICU) care.All patients received folic acid and vitamin B12 during treatment to support hematopoiesis.

Patients were followed every month till complete remission and then 3-monthly till 1 year. Clinical and lab parameters at last follow-up were classified based on Cerevance criteria [6] into 4 categories: No remission (NR), partial remission (PR), complete remission (CR) and continuous complete remission (CCR).

Statistical analysis: Chi square test and Student $t$ test (two tailed, unpaired) were used to compare variables between primary and secondary AIHA. $P$ value less than 0.05 was considered significant. SPSS version 20.0 was used for the analyses.

\section{RESULTS}

Data of 50 children [median (range) age at diagnosis, 36 months (1.5 months-17 years)] were analyzed. Commonest clinical feature at diagnosis was pallor $(100 \%)$ followed by fever $(68 \%)$ and jaundice $(60 \%)$. Hepatomegaly $(90 \%)$ was seen more often than splenomegaly $(38 \%)$.

Mean (SD) hemoglobin at presentation was 4.7 (1.6) $\mathrm{g} / \mathrm{dL}$. Out of 50 children, $72 \%$ children presented with very severe anemia (hemoglobin $<3 \mathrm{~g} / \mathrm{dL}, n=6$ ) or severe anemia (hemoglobin $3-6 \mathrm{~g} / \mathrm{dL} n=33,66 \%$ ); only 1 child had mild anemia ( $>9 \mathrm{~g} / \mathrm{dL})$. Admission to intensive care unit (ICU) was needed in $28 \%$ children. Leukocytosis (after correction for nucleated RBCs) was noted in 27 (54\%) patients, and leucopenia in 4 (8\%) patients. Only 3 children had thrombocytopenia at diagnosis. Reticulocytosis was seen in 37 cases (74\%), whereas reticulocytopenia was seen in 13 cases $(26 \%)$. Elevated lactate dehydrogenase (LDH) was seen in $86 \%$ children with median (range) LDH level of 521.5(163- 12858) U/L.

Direct anti-globulin test was $4+$ positive in 26 children, $3+$ positive in 11 children, $2+$ positive in 5 children, and $1+$ positive in 5 children.In three DAT- negative children, the diagnosis was based on clinicopathological suspicion after ruling out other causes of hemolytic anemia and on the basis of response to treatment. Two out of three DAT-negative patients were positive for Donath Landsteiner test. Monospecific DAT test was performed in 24 children after its availability from the year 2013; of which, $\operatorname{IgG} \pm \mathrm{C} 3$ was present in $10(41 \%)$ children, IgM and $\mathrm{C} 3$ were present in 3 children (13\%) and both $\operatorname{IgG}$ and $\operatorname{IgM}$ with $\mathrm{C} 3$ were present in 4 children (17\%). Cold agglutinin testing was performed in 21 children and was positive in 13 children. Based on above results cold, warm, mixed AIHA and $\mathrm{PCH}$ was seen in $35 \%, 28 \%, 15 \%$ and $4 \%$ children, respectively. In the other $18 \%$ children seen prior to 2010, AIHA was unclassified.

Secondary AIHA was identified in $36 \%$ cases with etiology being infection in 5 (10\%) (M.pneumonia, 3; cytomegalovirus infection, 1; Plasmodium vivax malaria, 1), autoimmune diseases in $5(10 \%)$ (autoimmune hepatitis, 2; systemic lupus erythematosus (SLE, 2; and giant cell hepatitis, 1). Other causes leading to secondary AIHA were Evans syndrome (6\%); childhood malignancies $(6 \%)$ (Hodgkin lymphoma, 2 and precursor B cell acute lymphoblastic leukemia, 1); CVID, 1(2\%); and Wiskott Aldrich syndrome, 1 (2\%).

Among infants, hemolysis was found to be much severe than those who developed AIHA after infancy (mean (SD) hemoglobin, $3.96(1.18)$ vs. $5.13(1.65) \mathrm{g} / \mathrm{dL}$, $P=0.01$ ). In primary AIHA, the mechanism of hemolysis was more often IgM and combined antibody mediated than in children with secondary AIHA wherein it was mainly IgG-mediated hemolysis.

Steroids alone were used in 33 (66\%) children; other medications used were IVIg in 2 children, steroid and IVIg in 2, steroid and rituximab in 2, steroid, rituximab and cyclosporine in 1, and steroid and other drugs (three or more) in 7 children. Other immunosuppressive medications used were mycophenolate mofetil and azathioprine. Among three patients of Evan syndrome, two patients responded to first line glucocorticoid therapy and one responded to second line therapy with IVIg followed by rituximab. One patient improved spontaneously and was not given any therapy.

Treatment duration was longer for children with secondary AIHA than primary ( 11 vs 6.6 months, $P=0.02$ ) and in patients needing polytherapy than those who improved with steroids only (13.3 vs 7.5 months, $P=0.006$ ). Median (range) follow up duration was 73 (1$150)$ months; $29(58 \%)$ remained in CCR, and $16(32 \%)$ in CR (Fig.1). Despite relapse in 26\% cases, $61.5 \%$ still showed good response to steroids. One patient with 


\section{WHAT THIS STUDY ADDS?}

- Managing AIHA in infants and those with secondary AIHA is challenging, with almost one-third patients at our center needing additional agents to the steroid backbone.

secondary AIHA and underlying Hodgkin lymphoma died due to fulminant fungal sepsis and hemophagocytosis.

\section{DISCUSSION}

We present our institutional data on pediatric AIHA and underscore the preponderance of AIHA in younger children; although, the median age at diagnosis in our study was higher than that in previous studies (10.8-16 months) [6,7]. Patients younger than one year required ICU care in view of severe anemia and hypoxia, similar to the report by Fan, et al. [7].

In our study, $94 \%$ cases had positive DAT, similar to another Indian study by Naithani, et al. [8]. Negative DAT in some patients may be due to low titer of IgG antibodies or $\operatorname{IgA}$ or $\operatorname{IgM}$ auto antibody mediated hemolysis. Reticulocytopenia seen in $26 \%$ cases was probably due to destruction of erythroid progenitors by autoantibodies [9]. A French national study [6] also observed a high incidence of reticulocytopenia (39\%) indicating that although reticulocytosis is an important marker of hemolysis, its absence alone should not rule out AIHA.

We found that hemolysis was severe whenever combined or IgM coated antibody mediated hemolysis occurs. This observation was similar to previously published study by Sokol, et al. [10], which showed that compared to IgG mediated hemolysis alone, IgG along with IgM or IgA leads to more severe hemolysis. Secondary AIHA was due to infection in $10 \%$ whereas

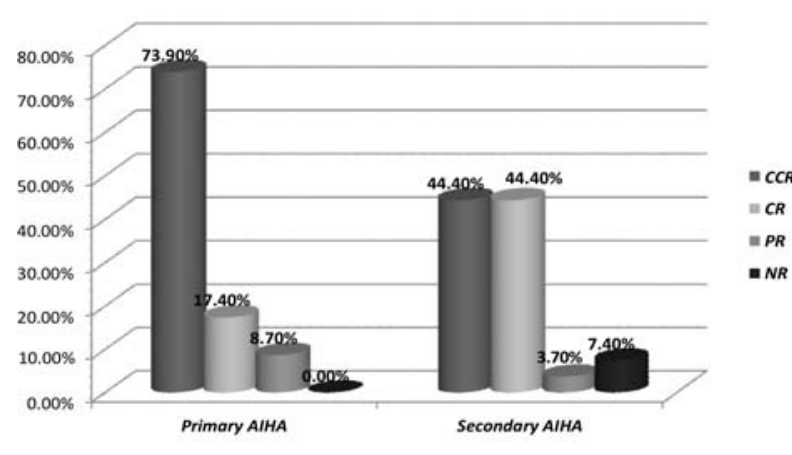

AlHA: autoimmune hemolytic anemia; CCR: continuous complete remission; $C R$ : complete remission; $P R$ : partial remission; NR-no remission.

Fig. 1 Comparison of remission status among primary and secondary autoimmune hemolytic anemia (AIHA).
Fan, et al. [7] showed that infection accounted for $97.6 \%$. In contrast to this, a French study [6] showed that secondary forms of AIHA were mainly due to immunological cause (53\%) and infections contributed to a very small portion (10\%). This observation may be due to increased burden of infection and early exposure of viruses like EBV in low or middle in-come countries.

Aladjidi, et al. [6] showed 90\% remission rates with $39 \%$ achieving CCR and 51\% attained CR. This may be due to prolonged usage of steroids (median duration 8 months). Two patients with $\mathrm{PCH}$ had early disease remission. This may be due to the self-limiting nature of the condition; however, as per unit policy they were also treated with short course of steroids. If there was no remission/steroid dependence with prednisolone in a dose of $0.2 \mathrm{mg} / \mathrm{kg} /$ day, second line treatment was used [11]. We needed immunosuppressants as second line of treatment in $26 \%$ cases. Rituximab was used in the standard dose of $375 \mathrm{mg} / \mathrm{m}^{2}$ per day [12].

Our study is limited by the fact that it is a retrospective study, comprises of a small cohort of patients and lacks protocol uniformity. Treating AIHA in children can be challenging and may need prolonged and complicated therapy, especially in secondary AIHA. We suggest that relapsed or refractory cases of AIHA should be cared by pediatric hematologists in a tertiary care center.

Ethics clearance: EC-SGRH; No.EC/09/20/1715, dated September 30, 2020.

Contributors: KBT: collection of data, analysis of data, writing of manuscript, revising it for important intellectual work; PS: collection of data, analysis of data, writing of manuscript; TP, DS: collection of data, analysis of data, writing of manuscript; AD, MK, AS: contributed patients, final editing of manuscript. All authors approved the final version of manuscript.

Funding: None; Competing interest: None stated.

\section{REFERENCES}

1. Chou ST, Schreiber AD. Autoimmune hemolytic anemia. In: Orkin SH, Fisher DE, Look AT, et al., editors. Nathan and Oski's Hematology and Oncology of Infancy and Childhood. 8th ed. Saunders Elsevier; 2015. p. 411-30.

2. Vaglio S, Arista MC, Perrone MP, et al. Autoimmune hemolytic anemia in childhood: Serologic features in 100 cases. Transfusion. 2007;47:50-4.

3. Anderson D, Ali K, Blanchette V, et al. Guidelines on the use of intravenous immune globulin for hematologic 
conditions. Transfus Med Rev. 2007;21:S9-56.

4. Norton A, Roberts I. Management of Evans syndrome. Br J Haematol. 2006;132:125-37.

5. Pui $\mathrm{CH}$, Wilimas J, Wang W. Evans syndrome in childhood. J Pediatr. 1980;97:754-58.

6. Aladjidi N, Leverger G, Leblanc T, et al. New insights into childhood autoimmune hemolytic anemia: A French national observational study of 265 children. Haematologica. 2011;96:655-63.

7. Fan J, He H, Zhao W, et al. Clinical features and treatment outcomes of childhood auto immune hemolytic anemia: A retrospective analysis of 68 cases. J Pediatr Hematol Oncol. 2016;38:50-55.

8. Naithani R, Agrawal N, Mahapatra M, Kumar R, Pati HP, Choudhry V.P. Autoimmune hemolytic anemia in children. Pediatr Hematol Oncol. 2007;24:309-15.

9. Mangan KF, Besa EC, Shadduck RK, Tedrow H, Ray PK.
Demonstration of two distinct antibodies in autoimmune hemolytic anemia with reticulocytopenia and red cell aplasia. Exp Hematol. 1984; 12:788-93.

10 Sokol RJ, Hewitt S, Booker DJ, Bailey A. Red cell autoantibodies, multiple immunoglobulin classes, and autoimmune hemolysis. Transfusion. 1990;30:714-17.

11. Ladogana, S, Maruzzi M, Samperi P, Perrotta S, Vecchio GCD, Farrugggia P. Diagnosis and Management of Newly Diagnosed Childhood Autoimmune Haemolytic Anemia. Recommendations from the Red Cell Study Group of the Pediatric Haemato-Oncology Italian Association. Blood Transfus. 2017;15:259-67.

12. Ducassou S, Leverger G, Fernandes H, Chambost H, Bertrand Y, Armari-Alla C. Benefits of rituximab as a second-line treatment for autoimmune haemolyticanaemia in children: A prospective French cohort study. $\mathrm{Br} \mathrm{J}$ Haematol. 2017;177:751-58.

\section{CLIPPINGS}

Acute kidney injury in pediatric patients hospitalized with acute COVID-19 and multisystem inflammatory syndrome in children associated with COVID-19 (Kidney Int. 2021 Mar 3: S0085-2538(21)00268-4)

This retrospective study was conducted in patients 18 years of age or below admitted to study the incidence, clinical presentation and outcome of acute kidney injury (AKI) in children with COVID-19 and Multisystem Inflammatory Syndrome. Total 152 children were included, out of which 97 had acute COVID-19 and 55 had COVID -19 and MIS-C. AKI occurred in $11.8 \%$ of children with acute COVID-19 and MISC. They had increased WBC counts and lower serum albumin levels on admission and decreased intravascular volume and distributive/cardiogenic shock. In addition, pediatric COVID19-related AKI was associated with poor outcomes, such as increased PICU and hospital length of stay. Their limitations were small sample size, retrospective study design, and most COVID-19 and MIS-C patients had a basic metabolic panel on presentation and during their hospital stay to assess for AKI. Utilization of serum creatinine without urine output and backcalculation of baseline creatinine values may have also underestimated the incidence of AKI. Therefore, further research in larger cohorts is needed to characterize AKI risk factors in children with acute COVID-19 and MISC

\author{
Long term renal survival of pediatric patients with \\ I lupus nephritis (Nephrol Dial Transplant. 2021 Apr 7: \\ gfab152)
}

This retrospective study conducted from 2000 till 2020 at Hacettepe University, tried to find out the clinical presentation, treatment options and renal prognosis in children with lupus nephritis. Data collected from medical charts and electronic records of 53 lupus nephritis children who had kidney biopsy at diagnosis. Overall, 52\% had lupus nephritis (LN); class IV LN (54.7\%) was most common followed by class III LN (22.6\%). Around $77.3 \%$ and $73 \%$ of children received complete and partial remission at 6 and 12 months, respectively. Five-and ten-year renal survival rates were $92 \%$ and $85.7 \%$, respectively. This study demonstrated that male gender, failure to achieve remission within 1 year after induction treatment and requiring dialysis at the time of diagnosis were the best predictors of poor renal outcome. Limited sample sign, retrospective study design, and bias in starting CYC for patients with more severe disease were some of the limitations reported. Prompt recognition and aggressive management of paediatric LN are essential to achieve and maintain remission

Afsana Jahan

dr.afsanajahan@gmail.com 